\title{
Dopaminergic Enhancement of Cellular Adhesion in Bone Marrow Derived Mesenchymal Stem Cells (MSCs)
}

Si Chen ${ }^{1,2, \pi}$, Bing Bai ${ }^{1,3, \pi}$, Dong Joon Lee ${ }^{1}$, Shannon Diachina ${ }^{1}$, Yina $\mathrm{Li}^{1,4}$, Sing Wai Wong ${ }^{1}$, Zhengyan Wang ${ }^{5}$, Henry CTseng ${ }^{6}$ and Ching-Chang $\mathrm{Ko}^{1,4^{*}}$

${ }^{1}$ Oral and Craniofacial Health Sciences Research, School of Dentistry, University of North Carolina, USA

${ }^{2}$ Department of Orthodontics, Peking University School and Hospital of Stomatology, PR China

${ }^{3}$ Department of Prosthodontics, China Medical University School of Stomatology, PR China

${ }^{4}$ Department of Orthodontics, School of Dentistry, University of North Carolina, USA

${ }^{5}$ Department of Pediatric Dentistry, University of North Carolina at Chapel Hill, USA

${ }^{6}$ Duke Eye Center and Department of Ophthalmology, Duke University Medical Center, USA

"These authors contributed equally as first author

\begin{abstract}
Dopamine (DA) is a well-known neurotransmitter and critical element in the mussel adhesive protein that has gained increasing attention for its role in cellular growth enhancement in biomaterials, including cellular adhesion improvement. As the mechanism underlying this remains unclear, the objective of this study was to explore the effects of DA on the adhesion properties of bone marrow derived rat mesenchymal stem cells (rMSCs) using an hydroxyapatite-gelatin nanocomposite biomaterial and to test whether the effects are mediated through various endogenously expressed DA receptors. Primary rMSCs were pretreated with D1-like antagonist, D2-like antagonist, or a combination of these antagonists followed by treatment with $50 \mu \mathrm{M} \mathrm{DA}$ and cellular adhesion quantification at 0.5 , 1,2 and 4 hours post DA addition. DA was found to increase rMSC adhesion and spreading at the 0.5 hour time-point and the dopaminergic effect on cell adhesion was partially blocked by DA antagonists. In addition, the D1-like and D2-like antagonists appeared to have a similar effect on rMSCs. Immunofluorescent staining indicated that the rMSC spreading area was significantly increased in the DA treated group versus the control group. Treatment of the D1-like DA antagonists with DA revealed that the actin filaments of rMSCs could not connect the membrane with the nucleus. In summary, DA was found to enhance early rMSC adhesion partially via DA receptor activation.
\end{abstract}

Keywords: Dopamine; Biomimetic biomaterial; Mesenchymal stem cells; Cellular spreading; Cellular adhesion

\section{Introduction}

Cellular attachment is a complex event, affected by cellular characteristics, material surface chemistry, and environmental factors. When cells contact the material surface, they adhere to the surface by adhesion molecules including selectins, syndecans, cadherins and especially integrins [1]. Integrins are transmembrane heterodimers, which possess cytoplasmic domains to transduce signals from the binding surface to the cell and thereby play a key role in cellular adhesion [2]. Poor cellular adhesion onto biomaterials can lead to the incomplete or poor bone formation after implantation in vivo. Thus, various surface modification methods such as physical adsorption, chemical immobilization of bioactive molecules, and plasma treatment have been introduced to enhance cellular adhesion onto artificial surfaces [3-5]. However, these methods are largely limited in application due to lack of stability, the requirement for sophisticated procedures, and high equipment cost.

Recently, coating with polydopamine (PDA) has been introduced to improve surface wettability for cellular adhesion within tissue engineering research. Studies have shown that PDA coating can increase the attachment of various cell types including osteoblasts, endothelial cells, and fibroblasts [6-9]. Inspired by those PDA characteristics, Ko et al. developed a 3D composite structure using an amalgamation of PDA to make hydroxyapatite-gelatin calcium silicate with polydopamine crosslinking (HGCS-PDA). HGCS-PDA can be fabricated through 3D printing techniques with porosity control, which can provide more surface area for cellular attachment. This novel material also has nearly equivalent mechanical properties as compared to cortical bone, with the incorporation of DA shown to increase the mechanical strength by $30 \%$ [10]. The HGCS-PDA material likely interacts with cells by providing
PDA for cell adhesion and as DA released from the material induces cellular responses via receptor binding. The affinity of mesenchymal stem cells (MSCs) toward the biomaterial is an early event considered crucial for bone regeneration.

One possible mechanism driving this enhanced cellular attachment and proliferation on the PDA coating is likely the latent reactivity of PDA to various nucleophiles with amine or thiol groups [11], which extracellular matrix (ECM)-derived adhesion proteins and growth factors can be efficiently attached to $[12,13]$. In addition, significantly improved MC3T3-E1 cell adhesion through integrin on the surface of PDA-modified materials has been described [13-15] and there is evidence that a PDA coating can promote osteogenic differentiation via the focal adhesion kinase (FAK) cascade [16]. Inhibition of FAK has been shown to prevent the expression of ERK-dependent alkaline phosphatase activity in osteoblast-like cell lines, consequently preventing further osteogenic differentiation. Furthermore, reduction of FAK activity in the osteoblasts of osteoporotic or osteoarthritic patients has been indicated [17]. ECM responsiveness during osteogenesis is caused by the aggregation of the $\alpha 2 \beta 1$ and $\alpha \mathrm{V} \beta 3$ integrin transmembrane

${ }^{*}$ Corresponding author: Ching-Chang Ko, DDS, PhD, Department of Orthodontics, UNC School of Dentistry, 275 Brauer Hall, Campus Box \#7454, Chapel Hill, NC 27599-7450, USA, Tel: (919) 537-3191; Fax: (919) 537-3754; E-mail: Ching-Chang_Ko@unc.edu

Received July 24, 2017; Accepted August 03, 2017; Published August 10, 2017

Citation: Chen S, Bai B, Lee DJ, Diachina S, Li Y, et al. (2017) Dopaminergic Enhancement of Cellular Adhesion in Bone Marrow Derived Mesenchymal Stem Cells (MSCs). J Stem Cell Res Ther 7: 395. doi: 10.4172/2157-7633.1000395

Copyright: @ 2017 Ko CC, et al. This is an open-access article distributed unde the terms of the Creative Commons Attribution License, which permits unrestricted use, distribution, and reproduction in any medium, provided the original author and source are credited. 
receptors, which convey information from the ECM to the intracellular compartment and subsequently activate intracellular signaling cascades such as FAK [18].

Another possible mechanism behind enhanced cellular attachment and differentiation may be the DA receptor mediated effect. Recent studies suggest that mesenchymal stem cells (MSCs) and osteoblasts expressed DA receptors and responded to DA agonists. For example, Shome $S$ et al showed that DA acted through the D2 receptors present in the MSCs to inhibit their mobilization to the wound beds [19]. In addition, MC3T3-E1 osteoblasts are known to express their functional DA receptors to enhance proliferation and mineralization in vitro [20]. These findings implicate that MSCs may respond in a receptor mediated manner to free DA monomers leaching out of PDA to stimulate their early adhesion via adhesion molecules. Still, whether the PDA coating itself can directly promote cell adhesion or if leached DA monomers are the driving factor behind cellular adhesion via the DA receptors remains unclear.

We have hypothesized that free DA, released from a biomaterial containing PDA crosslinking, promotes rat MSC (rMSC) adhesion by increasing integrin expression and activity. This type of material likely interacts with cells by providing PDA for cell adhesion and as DA released from the material induces cellular responses via receptor binding, with the affinity of rMSCs toward the biomaterial an early event considered crucial for bone regeneration. To test this hypothesis, we examined cell adhesion, cell spreading, and FAK activation to determine whether free DA can promote this integrin-mediated cell activity. The adhesion of rMSCs responding to both free and polymerized DA was investigated by checking the expression of five types of DA receptors and using a bone composite of hydroxyapatite-gelatin calcium silicate with PDA (HGCSPDA, the only bone biomaterial containing dopamine). The mechanism of rMSC adhesion was also examined by whether they were regulated by free DA and HGCS-PDA. The adhesion and spreading of rMSCs was illustrated through actin cytoskeleton formation and formation of cellmatrix adhesions at different time points. The expression of integrin genes was also identified. We found that free DA can enhance early cell adhesion and rMSC spreading by activating FAK-transduced ECM/ integrin signal pathways, suggesting that PDA-laced biomaterials could serve as a cytophilic surface during the cell seeding process in tissue engineered bone regeneration.

\section{Materials and Methods}

\section{Preparation of HGCS and HGCS-PDA coated dishes}

HGCS and HGCS-PDA materials were coated onto the surface of a $35 \mathrm{~mm}$ culture dish using the P-6000 spin coater (Specialty Coating Systems, Inc., Indianapolis, IN). The mixture of HGCS and HGCSPDA slurry was prepared as described previously [21]. Briefly, for HGCS coating, calcium hydroxide powder $(40.32 \mathrm{mg})$ was added to 1 $\mathrm{mL}$ of hydroxyapatite-gelatin (HAp-Gel slurry) and cross-linked with enTMOS [bis [3-(trimethoxysilyl)-propylethylenediamine, (Gelest, Inc., Morrisville, PA)]. After vigorous mixing, $50 \mu \mathrm{L}$ of the mixture was sprayed in the center of a spinning culture dish on the spin coater to form a thin, homogenous layer of coating. Each dish was spun for 20 seconds at 6,000 rpm. For the HGCS-PDA coating, DA (Acros Organics, New Jersey, NY) was added to the mixture of calcium hydroxide powder (40.32 mg) and $1 \mathrm{~mL}$ of HAp-Gel slurry. After crosslinking with enTMOS, DA was polymerized by interaction with $7.5 \%$ ammonium sulfate (Sigma-Aldrich, St. Louis, MO) to generate a HGCS-PDA mixture for culture dish coating, using the same method as the HGCS coating described above. The coated dishes were air-dried for 24 hours, sterilized under UV light for 72 hours, and washed with PBS before use.

\section{Contact angle measurement on the HGCS and HGCS-PDA coatings}

A contact angle was measured with a contact angle analyzer (CAM 200, KSV Instruments, Finland) to determine the hydrophilicity/ hydrophobicity of the HGCS and HGCS-PDA coated surface. Approximately $1 \mu \mathrm{L}$ of water was dropped onto the surface of the coated dishes and the contact angle was measured after a static time of 30 seconds. A total of three coated dishes from each material and five data points from each dish were used to calculate the mean and standard deviation (SD).

\section{DA release and measurement by ELISA}

To measure the DA release, ultra-pure water $(2 \mathrm{~mL})$ was added in HGCS and HGCS-PDA coated dishes. A test sample $(500 \mu \mathrm{L})$ was collected from each dish after vigorous mixing every 24 hours for 7 days. After the sample was collected, it was immediately added with $1 \mathrm{mM}$ EDTA and $100 \mathrm{mM} \mathrm{HCl}$ to stabilize dopamine and the $\mathrm{pH}$ was adjusted $(6.0 \pm 0.5)$. Collected samples were then stored at $4^{\circ} \mathrm{C}$ until use. DA concentrations were measured using an ELISA kit (DLD Diagnostika $\mathrm{GmbH}$, Hamburg Germany), following the manufacturer's instructions, and the optical density was measured at $450 \mathrm{~nm}$ in a microplate reader within 15 minutes.

\section{Isolation of rat bone marrow mesenchymal stem cells (rMSCs) and characterization}

The animal study was approved by the Institutional Animal Care and Use Committee (IACUC) at the University of North Carolina at Chapel Hill (approved protocol number 15-273). Sprague-Dawley rats (Charles River, Wilmington, MA, about $250 \mathrm{~g}$, 7 weeks old) were euthanized using carbon dioxide gas and cardiac puncture as the secondary method. The femurs were isolated aseptically and retrieved bone marrow by flushing with Dulbecco's modified Eagle's medium (DMEM, Invitrogen, Grand Island, NY, USA) supplemented with $20 \%$ fetal bovine serum (FBS, Equitech-bio, Kerrville, TX, USA), $250 \mu \mathrm{L}$ of GlutaMax (Invitrogen, Grand Island, NY, USA) and 2\% of penicillin/streptomycin (Invitrogen, Grand Island, NY, USA) after removal of the epiphysis at both ends. The suspension of all nucleated cells from the bone marrow was seeded into $100 \mathrm{~mm}$ culture dishes (Corning, NY, USA) and cultured at $37^{\circ} \mathrm{C}$ and $5 \%$ $\mathrm{CO} 2$. After three days, non-adherent cells were removed and adherent cells were cultured for 21 days in media refreshed every three days. When rMSCs reached approximately $80 \%$ confluency, the cells were passaged for further expansion. At passage 8 , the rMSCs were seeded at a density of $4.5 \times 10^{5}$ cells per $35 \mathrm{~mm}$ dish for adipogenic and osteogenic differentiation. To make rMSC aggregates for chondrogenesis, $2 \times 10^{6}$ cells per $15 \mathrm{~mL}$ conical tube were centrifuged at $1,200 \mathrm{rpm}$ for 3 minutes. Osteogenic differentiation was induced by replacing osteogenic media (growth media with $10 \mathrm{mM} \beta$-glycerophosphate, $0.2 \mathrm{mM}$ L-ascorbic acid, and $104 \mathrm{mM}$ dexamethasone) every 3 days for 21 days. Then, cells were fixed with $10 \%$ formalin and stained with Alizarin Red S for mineral nodule detection. Adipogenic differentiation was induced through use of adipogenic media (Stem Cell Technology, USA), replenished every 3 days for 14 days. Cells were then stained with Oil Red-O (Company info, USA). Chondrogenic differentiation was induced using 3D pellet cultures and replenishing chondrogenic media (Stem Cell Technology, USA) every 3 days for a total of 21 days. The aggregates were fixed in $10 \%$ formalin, embedded in optical cutting temperature (OCT), then sliced in $5 \mu \mathrm{m}$ sections using a cryostat. Safranin O staining was completed followed by fast green counter staining. For the single cell 
colony forming assay, $50 \mathrm{rMSCs}$ were seeded on a $35 \mathrm{~mm}$ dish. After 14 days, the culture dishes were fixed with $10 \%$ neutral formalin for 30 minutes and stained with $0.5 \%$ crystal violet solution for 10 minutes. All images were acquired using a light microscope (Nikon Eclipse Ti-U, Chiyoda-ku, Tokyo, Japan).

\section{RT-PCR and Western Blot analysis of DA receptor expression}

Total RNA extraction and RT-PCR (nested PCR) for DA receptor gene expression were performed as described previously [20], with primer sequences described in Table 1 . For western blots, a total of 20 $\mu \mathrm{g}$ of protein was used and separated using SDS-PAGE with GAPDH serving as the loading control. The detailed procedures for western blotting are described in the previous study [20].

\section{Cell adhesion and spreading assay}

After overnight starvation, rMSCs were incubated with/without the DA receptor antagonists $\mathrm{SCH} 23390$ (Tocris Bioscience, Bristol, UK), Eticlopride (Tocris Bioscience, Bristol, UK), GR103691 (Tocris Bioscience, Bristol, UK), and L741742 (Tocris Bioscience, Bristol, UK) with final concentrations of $1 \mathrm{mM}, 1 \mathrm{mM}, 0.5 \mathrm{mM}$ and $1 \mathrm{mM}$, respectively. After 30 minutes, DA $(50 \mu \mathrm{M})$ was added to the cells before seeding at a density of $2 \times 10^{4}$ per well in a 96-well plate that was pre-coated with type I collagen solution $(10 \mu \mathrm{g} / \mathrm{mL})$ and incubated at $4^{\circ} \mathrm{C}$ overnight. At $0.5,1,2$ and 4 hours, MTS [(3-(4, 5-dimethylthiazol-2-5-(3 carboxymethoxyphenyl)-2-(4-Sulfophenyl)2H-tetrazolium), (Promega, Madison, WI, USA)] assay was performed to quantify attached cells.

For the spreading assay, rMSCs were plated at $5 \times 10^{4}$ cells on each coverslip coated with type I collagen, HGCS, and HGCS-PDA, respectively. Collagen solution $(10 \mu \mathrm{g} / \mathrm{mL})$ was added on the coverslip placed in $35 \mathrm{~mm}$ dish and stored at $4^{\circ} \mathrm{C}$ overnight. rMSCs in the $\mathrm{DA}+\mathrm{D} 1$-like receptor blocker $(\mathrm{RB})$ group were first treated with the D1/5 receptor antagonist $\mathrm{SCH} 23390$ (Tocris Bioscience, Bristol, UK) at a final concentration of $1 \mathrm{mM}$. After incubation at $37^{\circ} \mathrm{C}$ for 30 minutes, both DA only and DA+D1-like RB group were treated with $50 \mu \mathrm{M}$ of DA. The rMSCs were directly seeded onto the coated dishes for $0.5,1$, 2 and 4 hours, respectively and then fixed with $4 \%$ paraformaldehyde (PFA). This was followed by permeabilization in $0.1 \%$ Triton X-100 (Sigma-Aldrich, St. Louis, MO) in phosphate buffered saline (PBS) for 5 minutes followed by blocking with $1 \%$ of bovine serum albumin (BSA: Gibco, Gran Island, NY, USA) for 30 minutes. Anti-Vinculin antibody (1:100) was incubated at $4^{\circ} \mathrm{C}$ overnight. After vigorous washing, Alexa Fluor ${ }^{\circledR} 488$ (Invitrogen Gran Island, NY USA) and TRITCconjugated phalloidin (Millipore, Billerica, MA USA) antibody (1:500) were incubated together at room temperature for 1 hour. Nuclei were counterstained with DAPI for 5 minutes. Antibodies were diluted using $1 \%$ BSA in PBS. The vinculin, F-actin phalloidin, and DAPI staining were observed with a Nikon fluorescence microscope with TRITC, FITC and DAPI filters and images were acquired with a digital camera (Nikon Instruments, Melville, NY USA). Cell numbers and spreading areas were measured with Image J software (National Institutes of
Health, Bethesda, MD USA). Ten random images from each slide ( $\mathrm{n}=5)$ were acquired for analysis for each condition.

\section{qPCR of cell adhesion related genes}

For qPCR analysis, rMSCs treated with $50 \mu \mathrm{M}$ DA were seeded on $35 \mathrm{~mm}$ culture dishes coated with type I collagen, HGCS, or HGCSPDA. Non-treated rMSCs served as a control. Integrin $\beta 1, \beta 3, \alpha 2, \alpha \mathrm{V}$ and FAK gene expression were measured using qPCR. Briefly, RNA was extracted using TRIzol Reagent (Invitrogen, Carlsbad, CA, USA) at 10, $15,30,45,60$ minutes after DA treatment. Then, cDNA was synthesized using iScript ${ }^{\mathrm{ms}}$ (BioRad Laboratories, Hercules, CA). The qPCR gene analysis was performed using the Applied Biosystems 7500 (Applied Bio-systems, Foster City, CA USA). PCR was performed for $1 \mathrm{~min}$ at $95^{\circ} \mathrm{C}$. A total of 40 amplification cycles were run, which consisted of 30 seconds denaturation at $95^{\circ} \mathrm{C}, 30$ seconds of annealing at $60^{\circ} \mathrm{C}$, and 30 seconds of extension at $72^{\circ} \mathrm{C}$. Samples were assayed in triplicate and the target gene expression levels were normalized in relation to the housekeeping gene GAPDH $(\mathrm{n}=3)$.

\section{Statistical analysis}

All experimental samples were carried out in triplicate. Quantitative data was represented as the mean \pm standard deviation (SD). Statistical
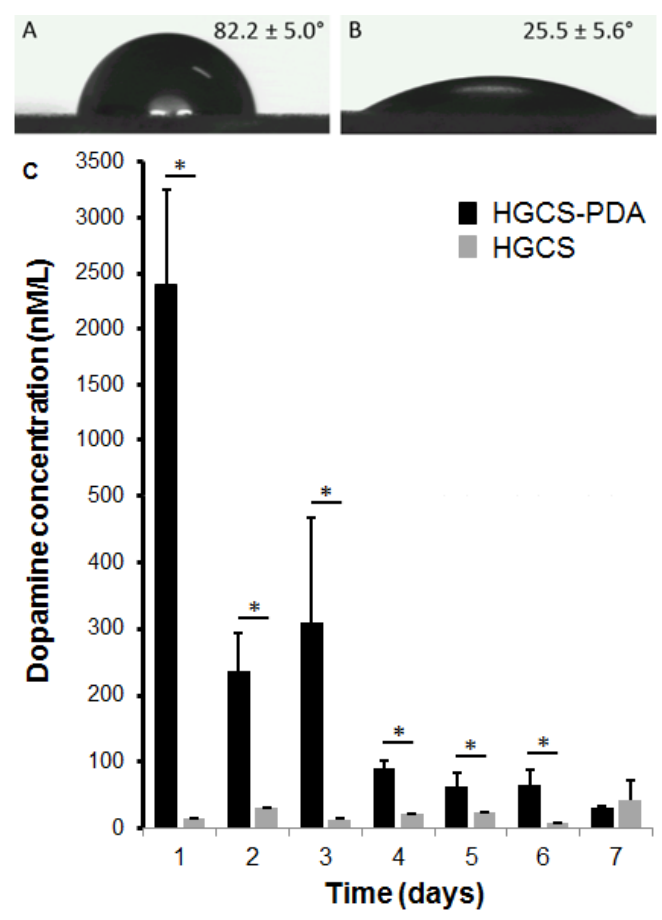

Figure 1: $\mathbf{A}$ and $\mathbf{B}$ Measurements of Water contact angle of dish surfaces coated with HGCS and HGCS-PDA, C amount of dopamine released from HGCS-PDA dish coating at different time points was measured by ELISA method.

\begin{tabular}{|c|c|c|}
\hline Gene & Forward primer sequence (5'-3') & Reverse primer sequence (5' - 3') \\
\hline Integrin 1 & CAAGTGCCATGAGGGAAATGG & ATTGGGATGATGTCGGGACC \\
\hline Integrin 3 & TCCTATGGAGACACCTGCGA & AGGTACAGTTCACCGCGTTT \\
\hline Integrin V & GGCTCCAGGGAGGAGTTCTA & CTTGGCCCAGACTCGGAAAT \\
\hline Integrin 2 & AGGGTACCATTCGCACCAAG & AGTTGAACCACTTGCCCCAA \\
\hline FAK & CTTAATCTGGCCAGGACGGT & 130 \\
\hline GAPDH & ACCCACGGCAAGTTCAACGG & ACTAAGCTTCCCCTGTGCTC \\
\hline
\end{tabular}

Table 1: Primer sequences used for qPCR in this study. FAK: focal adhesion kinase and GAPDH: glyceraldehyde 3-phosphate dehydrogenase. 
Citation: Chen S, Bai B, Lee DJ, Diachina S, Li Y, et al. (2017) Dopaminergic Enhancement of Cellular Adhesion in Bone Marrow Derived Mesenchymal Stem Cells (MSCs). J Stem Cell Res Ther 7: 395. doi: 10.4172/2157-7633.1000395

analysis was performed with a one-way analysis of variance (ANOVA); p-values less than 0.05 were considered statistically significant.

\section{Results}

\section{HGCS-PDA surface characteristics and release of DA}

Water contact angles in the HGCS-PDA group $\left(25.5 \pm 5.6^{\circ}\right)$ were lower than that in HGCS group $\left(82.2 \pm 5.0^{\circ}\right)$, indicating that the HGCSPDA surface has greater hydrophilicity than the HGCS surface (Figure $1 \mathrm{~A}$ and $1 \mathrm{~B}$ ). Free DA release from the coating was measured using ELISA and the peak value of leached free DA was detected and converted to concentration based on the standard curve, which was $2398.04 \pm 847.50$ $\mathrm{nmol} / \mathrm{L}$. This was significantly higher than control levels of $8.79 \pm 2.02$ $\mathrm{nmol} / \mathrm{L}$. DA concentration was found to decrease 75 -fold from day 1 to Day 7. The DA concentration at day 7 was $31.84 \pm 1.27 \mathrm{nmol} / \mathrm{L}$, which was similar to the HGCS group at day $7(42.74 \pm 30.49 \mathrm{nmol}$ per L) (Figure 1C).

\section{Expression of DA receptors in rMSCs}

rMSCs were isolated from the bone marrow of the Sprague Dawley rat (6 weeks old, male) according to the procedure in the previous study [22]. rMSCs were expanded to passage 5 and investigated for DA

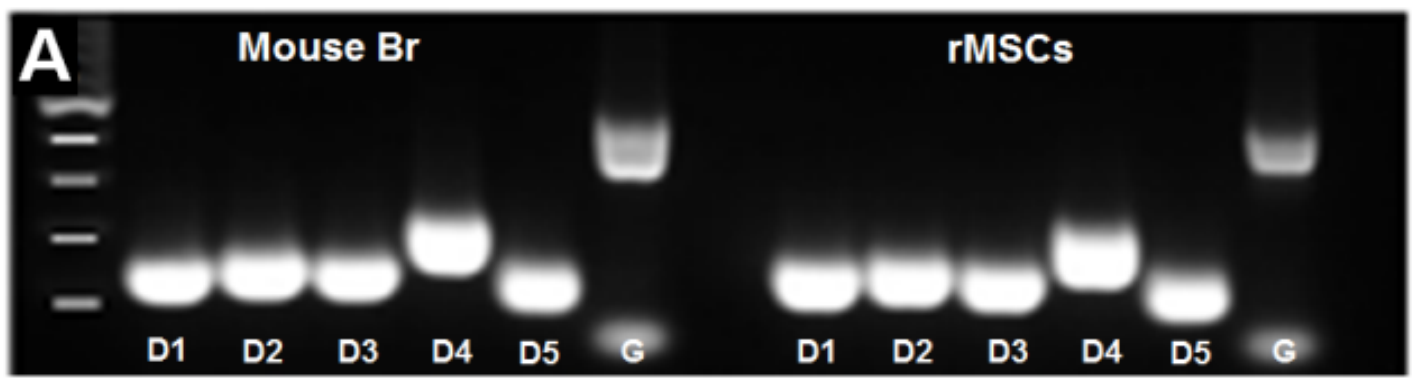

B $\mathrm{D}_{\text {1-like }}$ Family (D1 and D5)
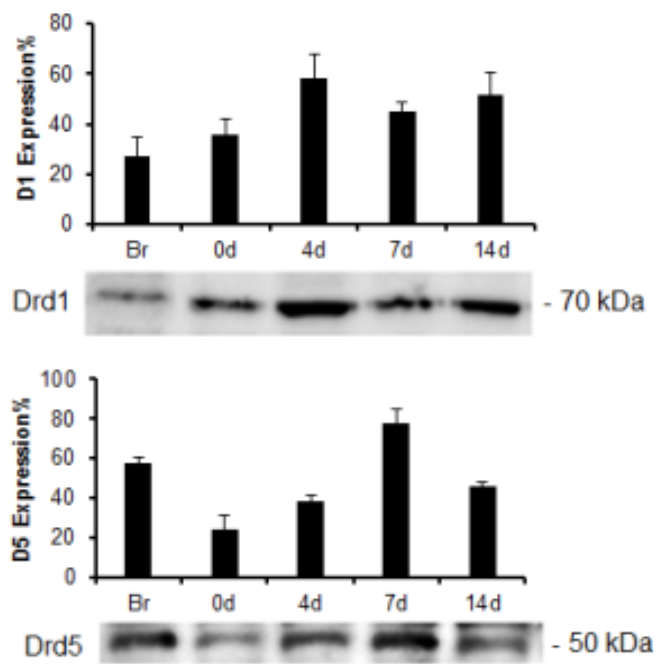

$\beta$-actin

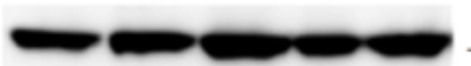

$.42 \mathrm{kDa}$

\section{$D_{2-\text { like }}$ Family (D2, D3 and D4)}
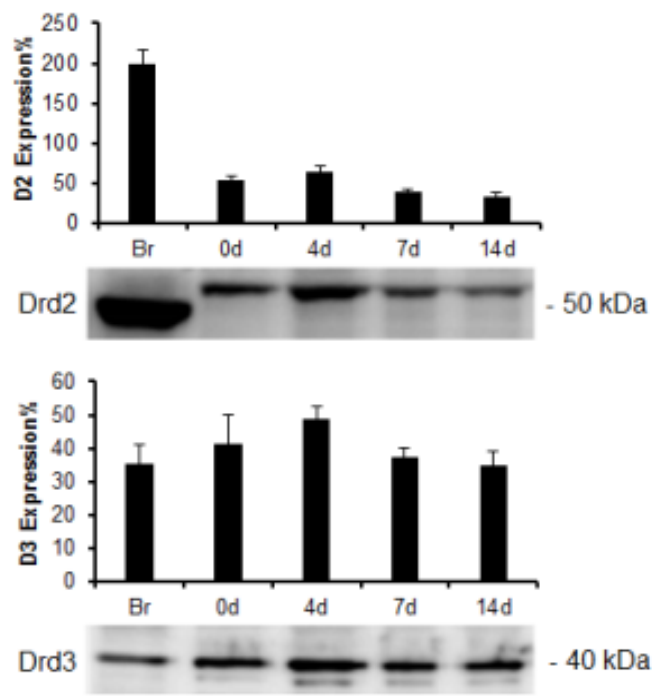

$-40 \mathrm{kDa}$

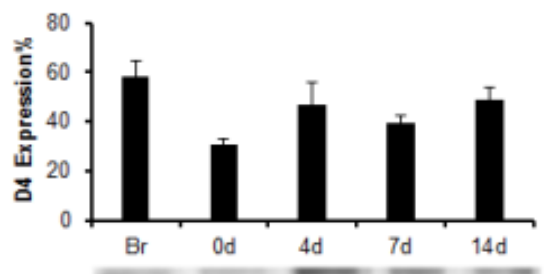

Drd4

Figure 2: A DA receptor expression detected by Nested PCR or western blot. DA receptor gene expression in cultured rMSCs was detected by Nested PCR. Mouse brain tissue were used as positive controls. B Western blot analysis of DA receptors on the rMSCs were evaluated during osteogenic differentiation at day $0,4,7$ and 14. Brain tissue protein was used as a control to identify the correct size of each DA receptor protein and binding specificity against each DA receptor antibody. Each sample was normalized by beta actin using Image $\mathrm{J}$ software. The relative molecular mass of significant bands identified on immunoblots was labelled on the right side. Br: brain, bp: base pair, G: GAPDH. 
receptor presence through use of PCR and western blotting. Results were compared to control lysates containing DA receptors expressed in the brain. PCR showed expression of all five DA receptor genes in rMSCs and their size were identical compared to the DA receptors genes of mouse brain (Figure 2A). Western blot analysis of rMSCs showed the presence of all five DA receptors in these cells. The bands for these receptors showed similar molecular weights as those in mouse brain (Figure 2B). The bands of five DA receptors, D1, D2, D3, D4, and D5 were detected around 70, 50, 50, 50 and $70 \mathrm{KDa}$ respectively in both mouse brain and rMSCs.

\section{rMSC adhesion and spreading}

To assess the effect of adding free DA on cell adhesion and spreading, we counted the number of cells attached to each substrate as well as their cell areas. rMSCs in the DA treated group indicated significantly higher cell adhesion (51 \pm 13 cells) at 30 minutes compared with the untreated control ( $45 \pm 12$ cells). The increased binding was blocked by $\mathrm{DA}+\mathrm{D} 1$-like receptor blocker $(\mathrm{RB})(28 \pm 4$ cells). Although there is no statistical difference in cell adhesion between the DA treated group and DA+D1-like receptor blocker group at 1,2 and 4 hours, blocking rMSCs with D1-like RB showed a significant decrease in the number of attached cells on collagen coating after 30 minutes (Figure 3A).

We also found DA has an effect on cell spreading, with rMSCs treated with DA indicating higher spreading area $(2108.7 \pm 567.1$ $\mu \mathrm{m}^{2}$ ) with more filopodia processes compared to cells in the control group $\left(1272.7 \pm 419.5 \mu \mathrm{m}^{2}\right)$ and $\mathrm{DA}+\mathrm{D} 1 / 5 \mathrm{RB}$ treated group $(1528 \pm$ $330.4 \mu \mathrm{m}^{2}$ ), which presented a smaller circular area with fewer or no cell processes evident at 30 minutes. No significant difference of rMSC spreading was found at 1,2 and $4 \mathrm{hrs}$ in DA treated group compared
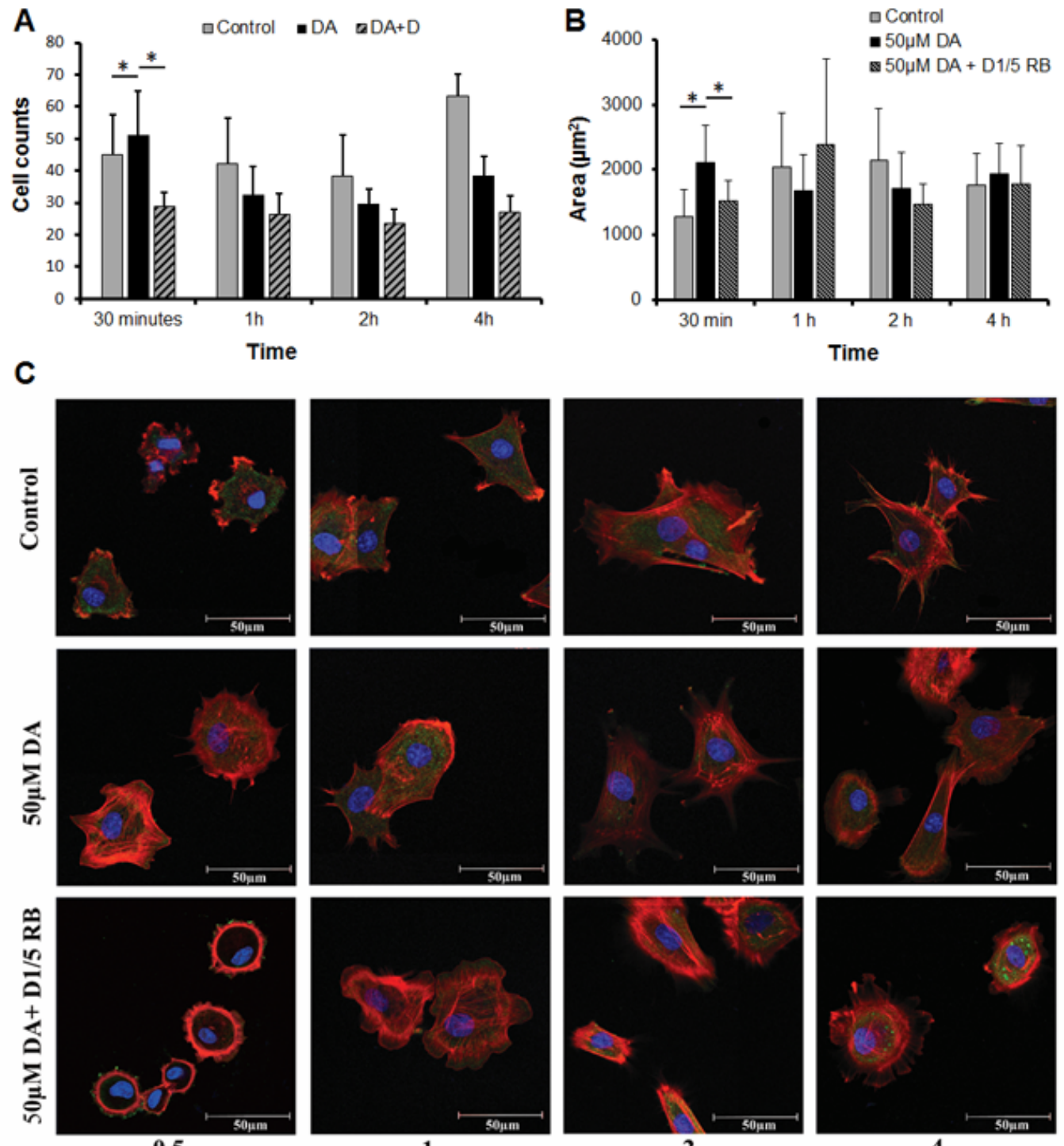

time (hour)

4

Figure 3: A Number of rMSCs on type I collagen coated plate treated without DA (controls), with DA, with DA+D1-like RB or DA+D2-like RB counted as cell number after $0.5,1,2$ and 4 hours, respectively and $\mathbf{B}$ the cellular area was measured as the area/cell using Image $\mathrm{J}$. C All images were taken by selecting ten random areas and data were represented as average \pm standard deviation $\left(n=3,{ }^{*} p<0.05\right)$. Immuno-fluorescent confocal images of cytoskeletal actin (red), vinculin (green) and nucleus (blue) for rMSCs were acquired using confocal microscope. 
Citation: Chen S, Bai B, Lee DJ, Diachina S, Li Y, et al. (2017) Dopaminergic Enhancement of Cellular Adhesion in Bone Marrow Derived Mesenchymal Stem Cells (MSCs). J Stem Cell Res Ther 7: 395. doi: 10.4172/2157-7633.1000395

Page 6 of 10

with 30 minutes (Figure $3 \mathrm{~B}$ and $3 \mathrm{C}$ ), likely as the spreading at 30 minutes already reached the maximum.

Consistent with results from free DA experiments, rMSCs directly cultured on HGCS coated dishes have round morphology. This indicated that the cells were poorly spread out on HGCS-coated surfaces. In strong contrast, the rMSCs projected area increased progressively over time grown on HGCS-PDA coating (Figure 4), similar to that on a collagen coated control group (Figure 3C). Counting the number of cells showed that there were more cells attached to HGCS-PDA coated dishes at 30 minutes and 1 hour than to HGCS and that this trend reversed at the 2 and 3 hour time points (Figure $4 \mathrm{~A}$ ). This is likely because DA has a short-term effect on cellular attachment. Measurement of cell areas confirmed no significant difference was found until 4 hours after seeding between HGCS $\left(1149.4 \pm 289.5 \mu \mathrm{m}^{2}\right)$ and HGCS-PDA $(1473.2$ $\pm 312.3 \mu \mathrm{m}^{2}$ ) groups (Figure 4B).

In this study, confocal images showed that both rMSCs cultured on HGCS-PDA and treated with free DA had a positive effect on cell spreading. Enhanced cell spreading by DA treatment showed larger area with greater formation of filopodia, lamellipodia and cellular extensions compared to the HGCS only or the DA antagonist blocked group (Figure 3C and 4C). In particular, cells seeded on HGCS-PDA coating exhibited enhanced spreading versus those treated with free $\mathrm{DA}$, due to the combined effect from free DA and the PDA in the material.

\section{Adhesion related gene expressions}

To evaluate whether cell adhesion receptor expression is affected by DA treatment, gene expression of integrin $\beta 1, \beta 3, \alpha 2, \alpha \mathrm{V}$ and FAK were measured by the qPCR. For the collagen coated group, the peak level of integrin $\beta 1$ activity in cells treated with $50 \mu \mathrm{M}$ DA was observed at 30 minutes, which is earlier than the 45 minutes observed in the "non-DA" control group, perhaps due to earlier binding via these integrin transmembrane receptors occurring from the addition of free DA. In contrast, no change in the expression of integrin $\beta 3$ was observed for both treatment groups. The expression of integrin $\alpha 2$ in $50 \mu \mathrm{M}$ DA treated group was significantly higher than in the control group without DA at 30 and 45 minutes. Significantly higher expression of integrin $\alpha \mathrm{V}$ was observed at 30 minutes, whereas significant higher expression of FAK was observed at 30, 45 and 60 minutes in $50 \mu \mathrm{M} \mathrm{DA}$ treated group than in the "non-DA" control group (Figure 5).

Cell adhesion receptor gene expression was also evaluated in cells coated on HGCS-PDA and HGCS coated groups. Significantly higher
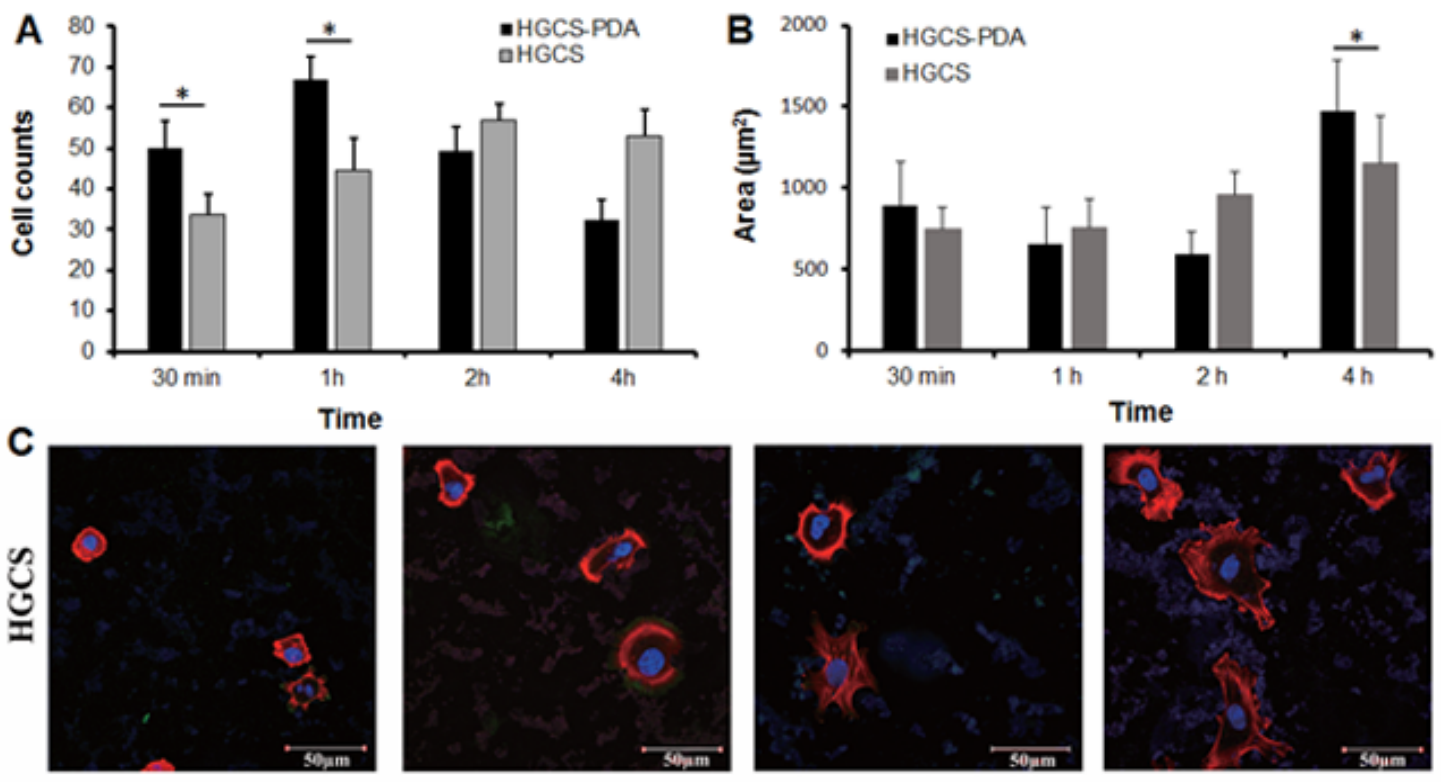

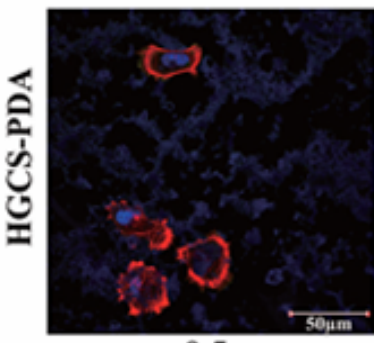

0.5

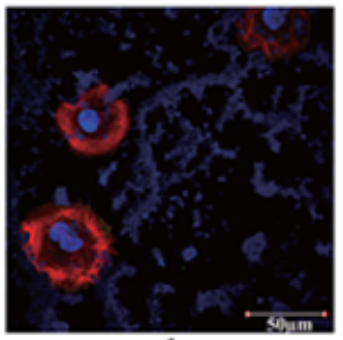

1

time (hour)

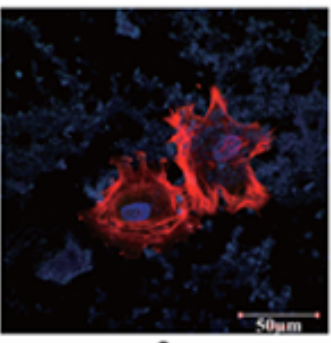

2

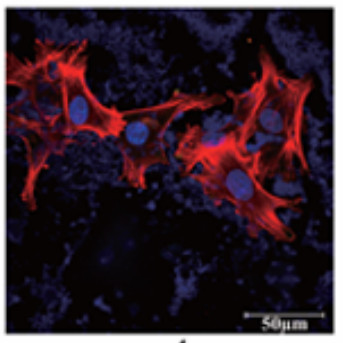

4

Figure 4: A Number of rMSCs on HGCS-PDA and HGCS coated coverslips was counted as cell number after $0.5,1,2$ and 4 hours, respectively and B the cellular area was measured as the area/cell using Image J. C Images were taken by selecting ten random areas and data were represented as average \pm deviation $\left(n=3,{ }^{*} p<0.05\right)$. Immuno-fluorescent images of cytoskeletal actin (red), vinculin (green) and nucleus (blue) for rMSCs were acquired using confocal microscope. 
expressions of integrin $\beta 1, \alpha 2, \alpha \mathrm{V}$ and FAK were detected in the HGCSPDA coated group at 30 minutes after seeding than in the HGCS coated group. While the peak of integrin $\beta 3$ expression occurred earlier at 10 minutes, no difference was found for other time points. Activity of integrin $\beta 1$ and FAK remained higher at 45 minutes in the HGCS-PDA coated group than the HGCS coated group (Figure 6). The differences in gene expression level between the HGCS-PDA or HGCS coating groups were more distinctive versus when cells were treated with free DA as shown in Figure 5. The peak level of integrin $\beta 3$ gene expression was evident at 10 minutes for the HGCS-PDA group, while the rest of the genes reached peak expression at 30 minutes.

\section{Discussion}

PDA coating as a surface modification has been reported to effectively improve cell attachment to materials without causing adverse effects on biological cell behaviors [23] and DA functional groups such as catechol and amine [18] may play key roles on the cell adhesion. Enhanced cell attachment to materials has been reported when a PDA coating has been utilized [23]. rMSC morphology was correlated with their physiological behavior and the shape was suggested to be a key regulator of rMSC commitment [24]. On rough surfaces, the cells were polygonal in shape with many thin filopodia for surface attachment [25]. Cell spreading increased osteoblast differentiation in pre-osteoblastic progenitors [26].

In this study, DA was incorporated into the HGCS-PDA biomaterial instead of coating on the surface to improve mechanical strength [27]. The HGCS-PDA can be fabricated through 3D printing techniques with excellent porosity control, which can provide greater surface area for cellular attachment. Among the numerous approaches to improve cell seeding on the biomaterial surface, the most common techniques
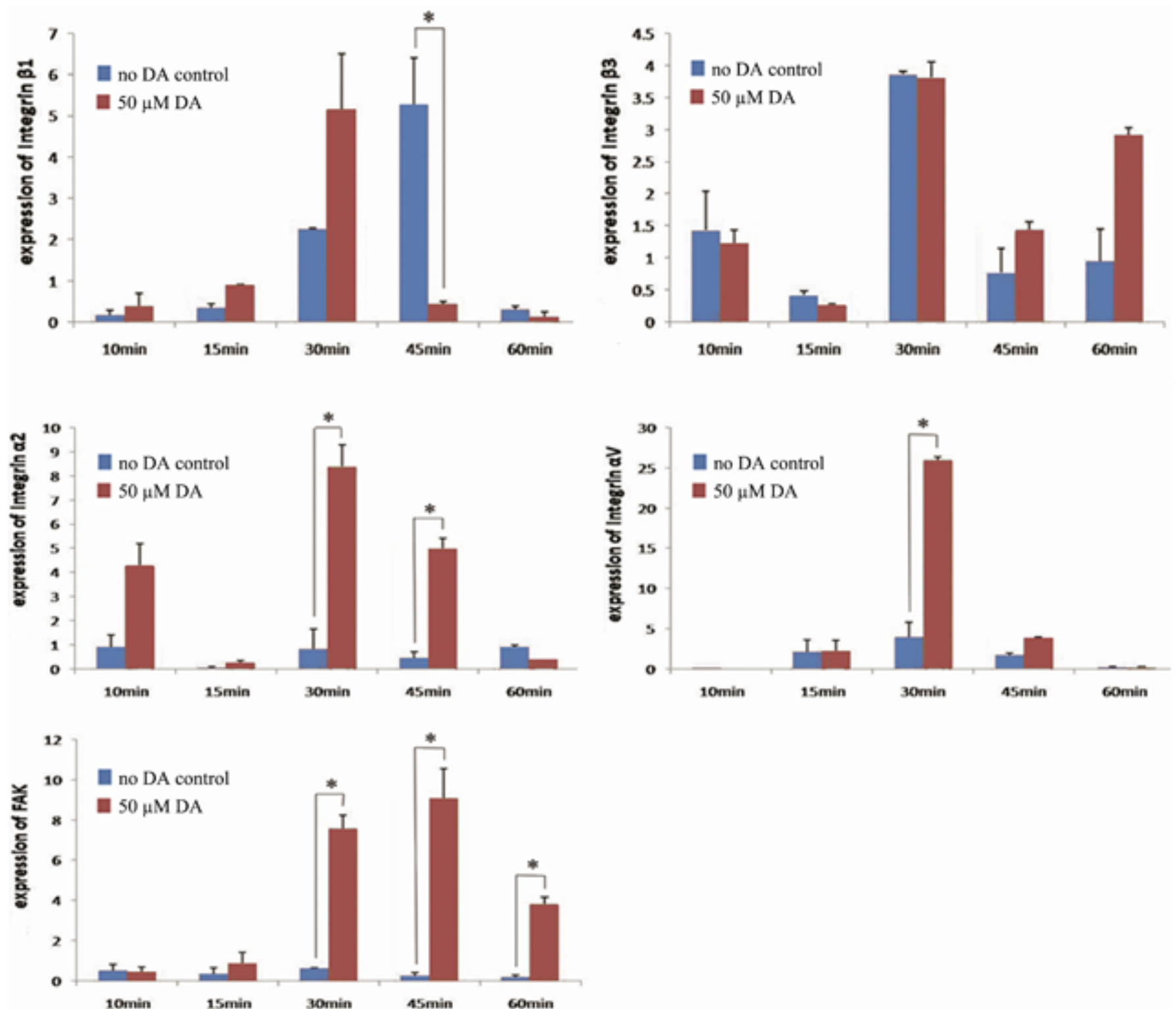

Figure 5: Gene expression of Integrin $\beta 1, \beta 3, \alpha 2, \alpha V$ and FAK in rMSCs treated with or without $50 \mu M$ dopamine (DA) at 10, 15, 30,45 and 60 minutes after seeding on type I collagen coated dishes $\left({ }^{*} p<0.05\right)$. 
involve the use of a biomolecular coating such as collagen, chondroitin sulfate, sulfated hyaluronan, diamines, heparin, and RGD motif [28]. However, these coating methods experience limitations in regard to practical applications due to lack of stability and the requirement for complicated or expensive procedures [29-31]. Thus, the HGCS-PDA material with DA incorporated can be a good alternative candidate for these conventional methods. Furthermore, water contact angle measurements revealed the addition of PDA greatly improved the hydrophilicity of HGCS for favorable cell attachment conditions.

We further asked whether contact between the DA substrate and cells or free DA released from the material and interacting with cells via DA receptors was responsible for the observed cellular effects. Free DA effect on rMSCs was analyzed through comparison among DA-treated, no DA treatment, and DA receptor antagonist treated groups on type I collagen coating. Additionally, the combined material effect was analyzed between the HGCS-PDA and HGCS coated groups. According to the previous study, $50 \mu \mathrm{M} \mathrm{DA}$ was verified to be a non-toxic and effective concentration in regard to MC3T3-E1 cell proliferation and osteogenic differentiation in vitro [20]. The same concentration was therefore used to treat rMSCs. Cell counting assays confirmed that significantly higher cell adhesion was achieved in the DA treated group (Figure 3A). Blocking with D1-like DA receptor blockers resulted in a significant decrease in cell adhesion at 30 minutes, while blocking with D2-like receptors had little or no effect on inhibiting cell adhesion. The suppressive effect on the rMSCs' adhesion resulting from D1-like RB returned to a level that was statistically higher than the control group at 4 hours, though the adhesion was lower than that of the unblocked DA group. This phenomenon can be explained by the time-related antagonistic effect. The DA treated group maintained significantly higher cell adhesion results within the four groups at all-time points. Overall, it was found that DA led to increased cell spreading and that adhesion can be blocked by DA receptor blockers, indicating that binding is receptor specific.
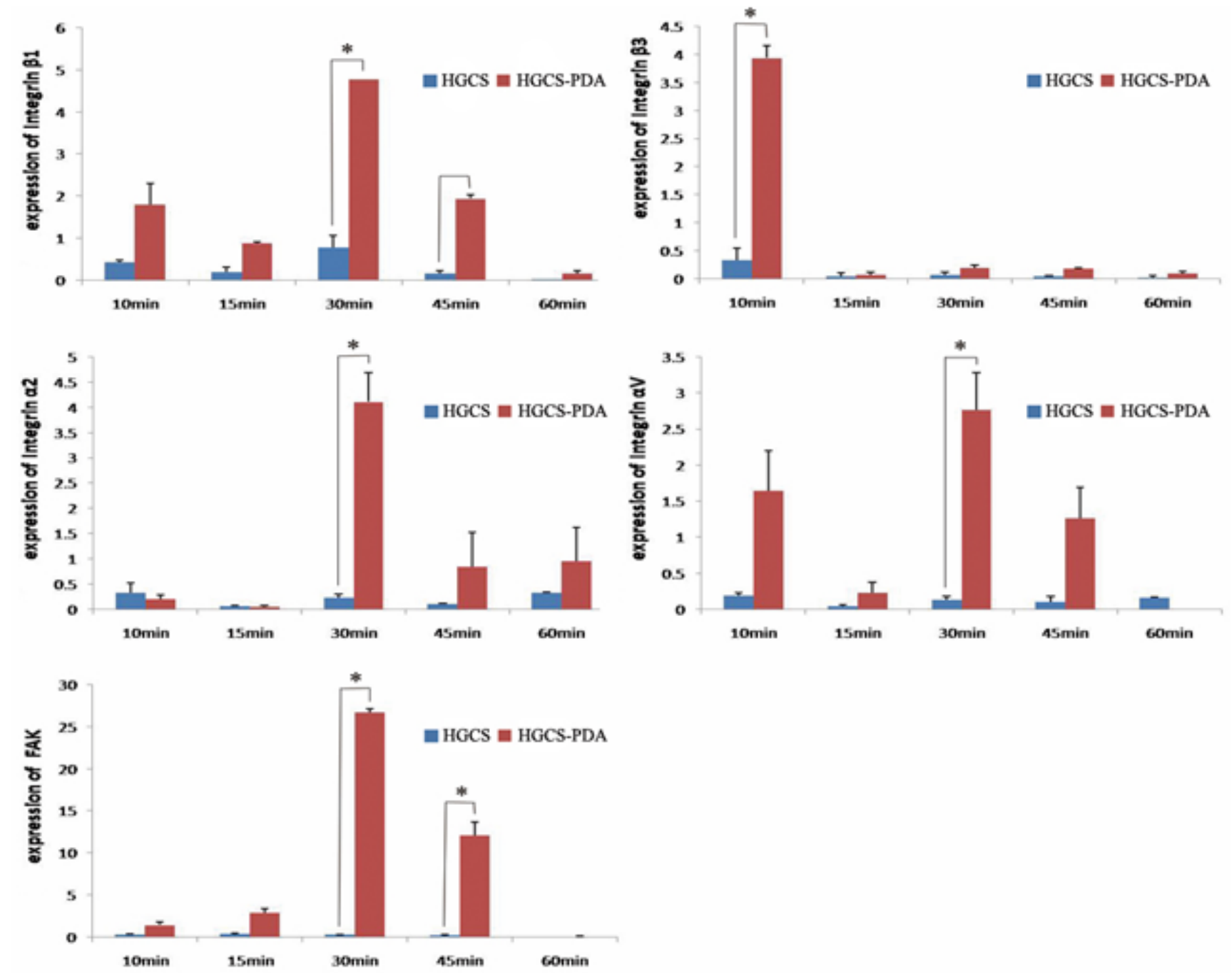

Figure 6: Gene expression of Integrin $\beta 1, \beta 3, \alpha 2, \alpha \mathrm{V}$ and FAK in rMSCs at 10, 15, 30, 45 and 60 minutes after seeding on HGCS-PDA or HGCS coated dishes $\left({ }^{*} \mathrm{p}<0.05\right)$. 
Formation of focal adhesions (FA) initiates the interaction between cells and substrate. Studies have suggested that rMSC gene expression can be induced by nanotopographical signals through FA and actomyosin cytoskeleton contractility [32]. FAK is one of the two major influencing agents in the FA processes activated by integrin-fibronectin interactions [33]. Matrix responsiveness during osteogenesis is caused by the $\alpha 2 \beta 1$ and $\alpha \mathrm{V} \beta 3$ integrin binding that subsequently activate intracellular signaling cascades [34]. Therefore, the expression levels of integrin $\beta 1, \beta 3, \alpha 2, \alpha \mathrm{V}$ and FAK genes were measured after free DA treatment and direct material interaction. The HGCS-PDA group demonstrated enhanced biological performance compared with HGCS, while the free DA treatment increased rMSC adhesion and cell spreading, though further studies are needed to verify the effect on later proliferation and osteogenic differentiation as well as for elucidation of the underlying mechanism. It remains unclear whether a DA receptor mediated pathway or other transmembrane receptor mediated pathways are involved. As the present study focused on the early stage of rMSC behavior, results are limited. Future studies should focus on the downstream targets of the integrin-FAK signaling complex (i.e. phosphorylation of FAK, expression of ERK), which is related to osteoblast differentiation. Additionally, the relationship between integrins and DNA methylation is a promising avenue of future investigation. Studies have shown that the a6 $\beta 4$ integrin has implications in cancer, predisposing the cell to a metastatic and invasive phenotype [35]. Integrins have been shown to affect DNA methylation at selected promoters, with DNA methylation playing a key role in downstream gene expression from the $a 6 \beta 4$ integrin [35] and in the regulation of integrin genes [36]. In this regard, histone modifiers such as DNA methyltransferases (DNMTs) and H3K9 methlyase G9a and GLP are critical players in establishing and maintaining DNA methylation in mammals [37]. These studies suggest that integrins have implications in cell motility, metastasis, and epigenetic mechanisms involving DNA methylation.

\section{Conclusions}

This study examined the effects of DA leaching from the HGCS-PDA and free DA on rMSCs for their adhesion and spreading characteristics. Our results indicate that DA can increase early adhesion and spreading of rMSCs, partially via activation of DA receptors. Furthermore, we found that cell adhesion related gene expression of rMSCs (Integrin and FAK) is upregulated by free or immobilized DA by interaction with HGCS-PDA. Further investigation is needed to elucidate the dopaminergic signaling pathway responsible for modulating rMSC cell adhesion and whether a PDA coating directly promotes cell adhesion. HGCS-PDA could be a preferred candidate for bone scaffolding materials as it improves MSC adhesion on the porous surface, which enhances the bone formation in vivo.

\section{Conflict of Interests}

The authors declare no conflict of interests.

\section{Acknowledgements}

The authors would like to acknowledge Yan-Ting Lee for data analysis and Huamin $\mathrm{Hu}$ for the technical support of contact angle measurement.

\section{References}

1. Buxton RS, Magee Al (1992) Structure and interactions of desmosomal and other cadherins. Semin Cell Biol 3: 157-167. [PubMed]

2. Giancotti FG, Ruoslahti E (1999) Integrin Signaling. Science.285: 1028-1033. [PubMed]

3. Meyers SR, Kenan DJ, Khoo X, Grinstaff MW (2011) Bioactive stent surface coating that promotes endothelialization while preventing platelet adhesion Biomacromolecules 12: 533-539. [PubMed]

4. Xu FJ, Wang ZH, Yang WT (2010) Surface functionalization of polycaprolactone films via surface-initiated atom transfer radical polymerization for covalently coupling cell-adhesive biomolecules. Biomaterials 31: 3139-3147. [PubMed]

5. Martins A, Pinho ED, Faria S, Pashkuleva I, Marques AP, et al. (2009) Surface modification of electrospun polycaprolactone nanofiber meshes by plasma treatment to enhance biological performance. Small 5: 1195-1206. [PubMed]

6. Tsai WB, Chen WT, Chien HW, Kuo WH, Wang MJ (2011) Poly(dopamine) coating of scaffolds for articular cartilage tissue engineering. Acta Biomater 7 : 4187-4194. [PubMed]

7. Ku SH, Ryu J, Hong SK, Lee H, Park CB (2010) General functionalization route for cell adhesion on non-wetting surfaces. Biomaterials 31: 2535-2541. [PubMed]

8. Shin YM, Lee YB, Shin H (2011) Time-dependent mussel-inspired functionalization of poly(L-lactide-co- $\varepsilon$-caprolactone) substrates for tunable cell behaviors. Colloids Surf B Biointerfaces 87: 79-87. [PubMed]

9. Ku SH, Park CB (2010) Human endothelial cell growth on mussel-inspired nanofiber scaffold for vascular tissue engineering. Biomaterials 31: 9431-9437. [PubMed]

10. Lee H, Dellatore SM, Miller WM, Messersmith PB (2007) Mussel-inspired surface chemistry for multifunctional coatings. Science 318: 426-430. [PubMed]

11. Lee H, Rho J, Holmburg K, Messersmith PB (2009) Facile conjugation of biomolecules onto surfaces via mussel adhesive protein inspired coatings. Adv Mater 21: 431-434. [PubMed]

12. Poh CK, Shi Z, Lim TY, Neoh KG, Wang W (2010) The effect of VEGF functionalization of titanium on endothelial cells in vitro. Biomaterials 31: 15781585. [PubMed]

13. Yang K, Lee JS, Kim J, Lee YB, Shin H, et al. ( 2012) Polydopaminemediated surface modification of scaffold materials for human neural stem cell engineering. Biomaterials 33: 6952-6964. [PubMed]

14. Tsai WB, Chena WT, Chien HW, Kuo WH, Wang MJ (2011) Poly (dopamine) coating of scaffolds for articular cartilage tissue engineering. Acta Biomaterialia 7: 4187-4194. [PubMed]

15. Ku SH, Lee JS, Park CB (2010) Spatial control of cell adhesion and patterning through mussel-inspired surface modification by polydopamine. Langmuir 26 : 15104-15108. [PubMed]

16. Rim NG, Kim SJ, Shin YM, et al. (2012) Mussel-inspired surface modification of poly (L-lactide) electrospun fibers for modulation of osteogenic differentiation of human mesenchymal stem cells. Colloids Surf B Biointerfaces 91: 189-197. [PubMed]

17. Perinpanayagam $H$, Zaharias $R$, Stanford C, Brand R, Keller J, Schneider G (2001) Early cell adhesion events differ between osteoporotic and nonosteoporotic osteoblasts. J Orthop Res. 19: 993-1000. [PubMed]

18. Takeuchi Y, Suzawa M, Kikuchi T, Nishida E, Fujita T, et al. (1997) Differentiation and transforming growth factor- $\beta$ receptor down-regulation by collagen- $\alpha 2 \beta 1$ integrin interaction is mediated by focal adhesion kinase and its downstream signals in murine osteoblastic cells. Journal of Biological Chemistry 272 29309-29316.

19. Shome S, Dasgupta PS, Basu S (2012) Dopamine regulates mobilization of mesenchymal stem cells during wound angiogenesis. PLoS One 7(2): e31682. [PubMed]

20. Lee DJ, Tseng HC, Wong SW, Wang Z, Deng M, et al. (2015) Dopaminergic effects on in vitro osteogenesis. Bone Res 3: 15020. [PubMed]

21. Chiu CK, Lee DJ, Chen H, Chow LC, Ko CC (2015) In-situ hybridization of calcium silicate and hydroxyapatite-gelatin nanocomposites enhances physical property and in vitro osteogenesis. J Mater Sci Mater Med 26: 92-106. [PubMed]

22. Lotfy A, Salama M, Zahran F, Jones E, Badawy A, et al. (2014) Characterization of Mesenchymal Stem Cells Derived from Rat Bone Marrow and Adipose Tissue: A Comparative Study. Int J Stem Cells 7: 135-142. [PubMed]

23. Ku SH, Park CB (2010) Human endothelial cell growth on mussel inspired nanofiber scaffold for vascular tissue engineering. Biomaterials 31: 9431-9437. [PubMed]

24. McBeath R, Pirone DM, Nelson CM, Bhadriraju K, Chen CS (2004) Cell 
Citation: Chen S, Bai B, Lee DJ, Diachina S, Li Y, et al. (2017) Dopaminergic Enhancement of Cellular Adhesion in Bone Marrow Derived Mesenchymal Stem Cells (MSCs). J Stem Cell Res Ther 7: 395. doi: 10.4172/2157-7633.1000395

Page 10 of 10

shape, cytoskeletal tension, and rhoA regulate stem cell lineage commitment. Developmental Cell 6: 483-495. [PubMed]

25. Zhao G, Raines AL, Wieland M, Schwartz Z, Boyan BD (2007) Requirement for Both Micron and Submicron Scale Structure for Synergistic Responses of Osteoblasts to Substrate Surface Energy and Topography. Biomaterials 28: 2821-2829. [PubMed]

26. Yourek G, Hussain MA, Mao JJ (2007) Cytoskeletal changes of mesenchyma stem cells during differentiation. ASAIO J 53: 219-228. [PubMed]

27. Ko CC, Wang Z, Tseng HC, Lee DJ, Guez C (2014) Design, synthesis, and evaluation of polydopamine-laced gelatinous hydroxyapatite nanocomposites for orthopedic applications. Advance Bioceramics Biotechnologies II: Ceramic Trans 247: 135-148.

28. Wua S, Liua X, Yeungb K, Liud C, Yangd X (2014) Biomimetic porous scaffolds for bone tissue engineering. Material Science Engineering 80: 1-36.

29. Yuan S, Xiong G, Wang X, Zhang S, Choong C (2012) Surface modification of polycaprolactone substrates using collagen-conjugated poly(methacrylic acid) brushes for the regulation of cell proliferation and endothelialisation. Journal of Material Chemistry 22: 13039-13049.

30. Hsu SH, Lin CH, Tseng CS (2012) Air plasma treated chitosan fibers-stacked scaffolds. Biofabrication 4: 015002. [PubMed]

31. Inanc B, Elcin AE, Elcin YM (2006) Osteogenic induction of human periodontal ligament fibroblasts under two- and three-dimensional culture conditions. Tissue Engineering 12: 257-266.

32. Teo BK, Wong ST, Lim CK, Kung TY, Yap CH, et al. (2013) Nanotopography modulates mechanotransduction of stem cells and induces differentiation through focal adhesion kinase. ACS Nano 7: 4785-4798. [PubMed]

33. Deligianni DD (2014) Multiwalled carbon nanotubes enhance human bone marrow mesenchymal stem cells' spreading but delay their proliferation in the direction of differentiation acceleration. Cell Adh Migr 8: 558-562. [PubMed]

34. Xiao GZ, Gopalakrishnan R, Jiang D, Reith E, Benson MD, et al. (2002) Bone morphogenetic proteins, extracellular matrix, and mitogen-activated protein kinase signaling pathways are required for osteoblast-specific gene expression and differentiation in MC3T3-E1 cells. J Bone Miner Res 17: 101-110. [PubMed]

35. Chen M, Sinha M, Luxon BA, Bresnick AR, O'Connor KL (2009) Integrin alpha6beta4 controls the expression of genes associated with cell motility, invasion, and metastasis, including S100A4/metastasin. J Biol Chem 284: 1484-1494. [PubMed]

36. Uhm KO, Lee JO, Lee YM, Lee ES, Kim HS, et al. (2010) Aberrant DNA methylation of integrin alpha4: a potential novel role for metastasis of cholangiocarcinoma. J Cancer Res Clin Oncol 136: 187-194. [PubMed]

37. Zhang T, Termanis A, Ozkan B, Bao XX, Culley J, et al. (2016) G9a/GLP complex maintains imprinted DNA methylation in embryonic stem cells. Cell Rep 15: 77-85. [PubMed] 